\title{
Finite Element Analysis Of The Precracked Line Scratch Test
}

\author{
A.A. Volinsky", L. Mercado*, V. Sarihan", and W.W. Gerberich ${ }^{* *}$ \\ "Motorola, Digital DNA ${ }^{\mathrm{TM}}$ Labs, Semiconductor Product Sector, AZ \\ ** University of Minnesota, Dept. of Chem. Eng. and Materials Science, Minneapolis, MN.
}

\section{ABSTRACT}

In MEMS packages and silicon devices, the adhesion of interconnects to the substrate is a critical reliability issue. A Precracked Line Scratch Test (PLST) is among one of the available tests to measure the thin line adhesion. In the test, an initial crack is introduced at the interface between the thin line and the substrate. The line is then loaded from the precracked end. The load is recorded continuously while the crack propagates before and after the line buckles. This precracked line scratch test has been applied earlier to tungsten thin lines on silicon wafers [1]. A macroscopic version of the test was also performed to evaluate the analytical model [2]. In the macroscopic tests, polycarbonate lines were bonded to steel substrates with cyanoacrylate.

In this paper, finite element analysis is performed for the Precracked Line Scratch Test before line buckling. The energy release rates and phase angles are calculated based on the corresponding load and crack length. The results are then compared to the closed-form solution. Macroscopic experimental model along with the finite element solution has provided a way to derive the interface fracture toughness as a function of the crack length based on the load and crack length history. With the analysis in place, the precracked line scratch test can be used conveniently to study the adhesion of interconnects to passivation layers, MEMS devices and packages on different scales.

\section{INTRODUCTION}

Thin film adhesion is a very important property not only for the microelectronics and magnetic recording industries, but also for emerging technologies such as data transmission through optical switches dependent on microelectromechanical systems (MEMS). Films that adhere well to the substrate are desired. However, spontaneous delamination may occur due to crack growth between thin film and the substrate.

This paper considers the Precracked Line Scratch Test (PLST) for quantitatively measuring the practical work of adhesion of thin interconnect lines. The technique is extremely useful since it is applied to the as-deposited, as-processed lines, without changing the interfacial chemistry and film microstructure/properties.

Most qualitative adhesion tests empirically infer the adhesive strength by subjecting the specimen to some external load and measuring the critical value at which it fails [3]. While still useful for routine quality control, these tests do not measure the interface fracture toughness $\Gamma_{i}$, since the strain energy release rate usually can't be deconvoluted from the work of the external load. There are several qualitative adhesion tests such as the scotch tape or the peel test $[4,5]$. These tests are usually easy and fast to perform, but they do not provide accurate values of adhesion. In the scotch tape test for example a piece of adhesive tape is attached to the tested film and then pulled off at $180^{\circ}$. "Bad" adhesion is indicated if the film is removed with the tape: conversely, "good" adhesion is indicated if the film remains attached to the substrate. The ideal test should simulate the practical situation as closely as possible, while also being able to extract 
the value of strain energy release rate. If the actual structure is to experience only low service temperature upon fabrication, using high homologous temperature processing steps in test specimen preparation, such as diffusion bonding, is not desirable, since it severely alters interface adhesion properties [6]. Fracture mechanics consists of methods of mechanical calculations to assess the crack driving force (strain energy release rate), $G$, experimental measurements of $\Gamma_{i}$ (the materials resistance to crack growth), and of understanding $\Gamma_{i}$ by materials science.

Most of the quantitative test methods measure adhesion by delaminating thin films from the substrate. While debonding from the substrate, the thin film and/or the substrate usually experience plastic deformation, so it is difficult to extract the true adhesive energy from the total amount of energy dissipated. What is measured is the practical work of adhesion, or interfacial toughness:

$$
W_{A, P}=W_{A}+U_{f}+U_{s}+U_{\text {fric }}
$$

where $U_{f}$ and $U_{s}$ are the energies per unit area spent in plastic deformation of the film and the substrate respectively, and $U_{\text {fric }}$ are the energy losses due to friction. Although the last three terms appear to be simply additive, it should be noted that both $U_{f}\left(W_{A}\right)$ and $U_{s}\left(W_{A}\right)$ are functions of the true work of adhesion [7] and in many cases $U_{\text {fric }}\left(W_{A}\right)$ will be as well.

M. de Boer, et al. adjusted the original scratch test for fine line structures $[1,8,9]$. A thin metal line on a substrate is pushed with the asymmetric diamond wedge from its end. The thin line has a processed precrack in the form of a carbon layer, which makes it a valid fracture mechanics specimen. The precrack portion of the line is deformed elastically in the beginning of the test until the crack propagates. When the crack reaches its critical buckling length at a certain critical load, $P_{c r}$, the film buckles.

The test is applicable to the hard lines, capable of bearing a load to the crack tip without plastically deforming; it was originally carried out on thin $\mathrm{W}$ lines on oxidized silicon wafers.

\section{EXPERIMENT}

The Precracked Line Scratch Test has been modeled using the macroscopic setup of a

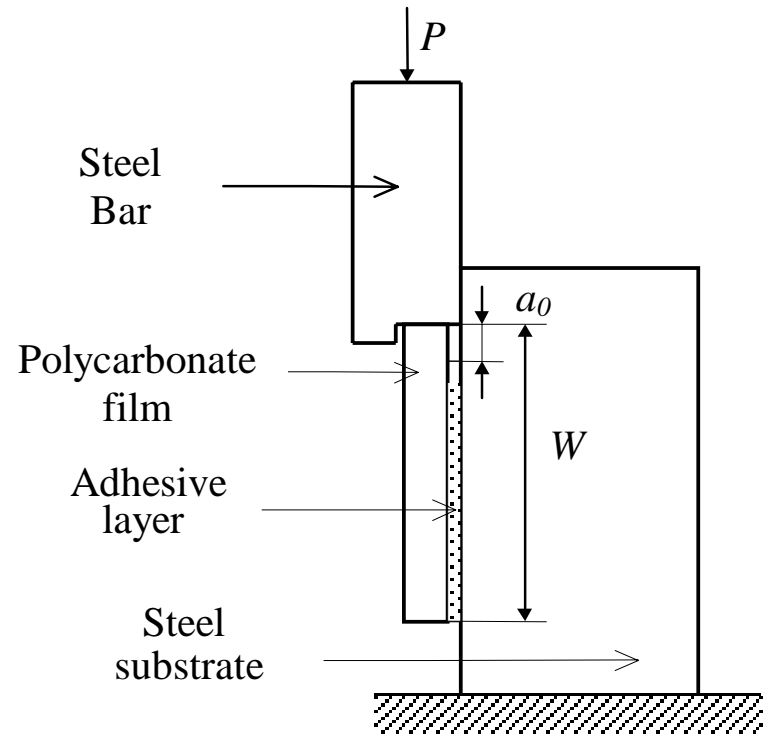
polycarbonate line bonded to steel with cyanoacrylate [2] (Figure 1).

Polycarbonate/cyanoacrylate conbination is suitable for in-situ crack length measurements since both materials are transparent. A $1.7 \mathrm{~mm}$ thick, $18 \mathrm{~mm}$ wide by $100 \mathrm{~mm}$ long line was bonded with cyanoacrylate to a 1015 steel substrate mechanically polished to 400 grit. A $10 \mathrm{~mm}$ "precrack" was formed by inserting a Teflon tape between the polycarbonate line and the steel substrate prior to bonding. All tests were performed using an Instron 8500 servo-hydrolic testing machine operating at room temperature normal conditions. Tests were video recorded and the crack length was measured from the video footage at multiple time steps.

Figure 1. Macroscopic PLST model. 


\section{PLST MECHANICS}

For a small initial crack during initial loading the precracked part of the line is deformed elastically:

$$
\varepsilon \approx \frac{\Delta}{a}
$$

where $a$ is the crack length, $\Delta$ is the absolute strain (Figure 2).

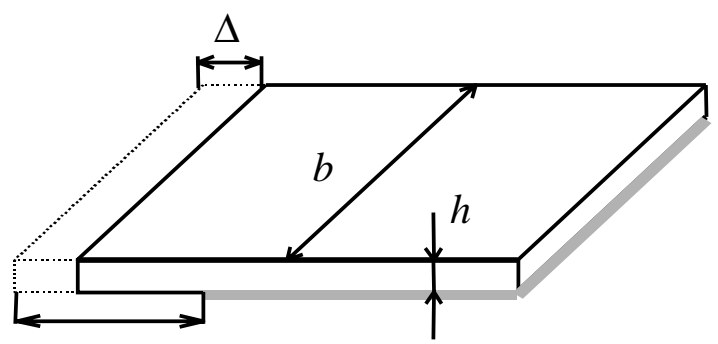

Figure 2. Precracked line mechanics schematic.

$a$

At any given moment prior to buckling the elastic energy stored in the line could be expressed as:

$$
U=\frac{\sigma \varepsilon}{2} a b h=\frac{\varepsilon^{2} E^{\prime}}{2} a b h
$$

where $E^{\prime}$ is the plane strain elastic modulus $\left(E^{\prime}=E /\left(1-v^{2}\right)\right), b$ and $h$ are the line width and thickness respectively (Figure 2). Derivative of the elastic strain energy with respect to crack length $a$ is:

$$
\frac{\partial U}{\partial a}=-\frac{\Delta^{2} E^{\prime} b h}{2 a^{2}}=-\frac{\varepsilon^{2} E^{\prime} b h}{2}
$$

The strain energy release rate $G$ is:

$$
G=-\frac{1}{b} \frac{\partial U}{\partial a}=+\frac{1}{b} \frac{\sigma^{2} E^{\prime} b h}{2 E^{\prime 2}}=\frac{\sigma^{2} h}{2 E^{\prime}}
$$

Now using the definition of stress $\sigma=$ load/area one can get the expression for $G$ prior to buckling [9] using the load, $P$ :

$$
G_{\text {pre-buckling }}=\frac{\sigma^{2} h}{2 E^{\prime}}=\frac{P^{2}}{2 b^{2} h E^{\prime}}
$$

Note that the crack length is not in the equation and the only experimental variable here that varied with the crack length is the load. This is important in terms of the experiment simplicity, since it may not be suitable to measure the crack length for the microscopic tests. This analysis is valid for the crack length greater than the line thickness.

Post-buckling solutions for the strain energy release rate are provided in [9, 10 and 2]. Crack length information is required in this case. As derived in [2], strain energy release rate, G after buckling is expressed:

$$
G_{\text {post-buckling }}=-\frac{1}{b} \frac{\partial U}{\partial a}=\frac{\pi^{2} E_{f}^{\prime} h^{3}}{24 a^{4}}\left(4 \delta a-\pi^{2} h^{2}\right)
$$

Now the stain energy release curve can be constructed throughout the whole test, before and after the line buckling (Figure 3). Prior to the line buckling an R-curve behavior is observed, 
when the strain energy release rate increases with the crack length due to increased plasticity at the crack tip. Based on the simple plane strain plastic zone size estimates [11], the strain energy release rate increase from 20 to $1000 \mathrm{~J} / \mathrm{m}^{2}$ corresponds to the plastic zone size increase from about 1 to $50 \mu \mathrm{m}$, which is still contained within the adhesive layer.

At the point of buckling there is a non-equilibrium crack growth, since the strain energy release rate, $G$, exceeds interfacial fracture toughness, $\Gamma(\psi)$ (Figure 4). This situation is analogous to the circular blister buckling [12]: at a certain level of stress, $\sigma_{\text {buckle }}$, and a certain crack length, $a_{1}$, line starts to buckle, at which point the interfacial fracture toughness drops under the influence of the phase angle decrease. The crack arrests at $a_{2}$ when the interfacial

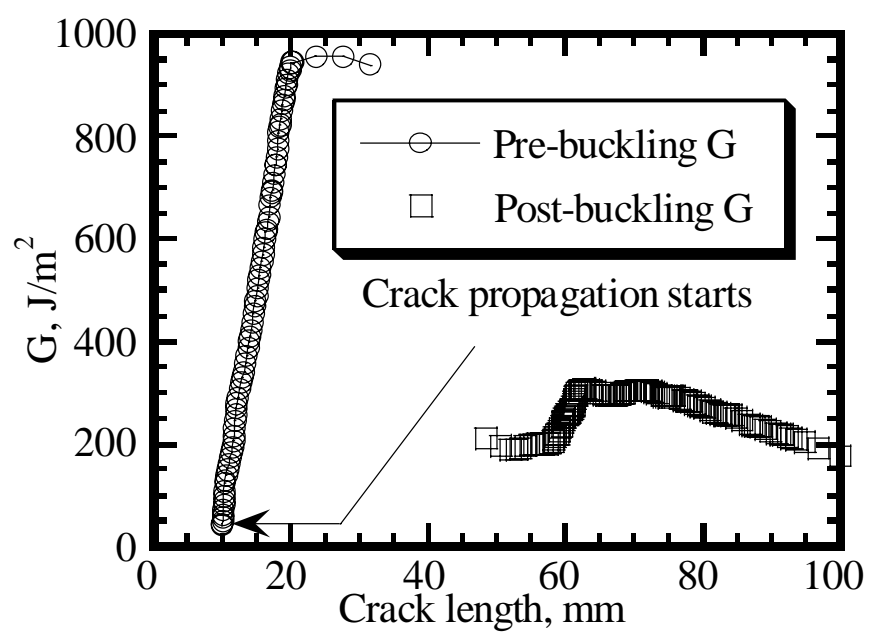
fracture toughness exceeds the strain energy release rate. At this point fracture is dominated by the Mode I stress component, and continues to grow stably until the total line decohesion [2]. For the PLST the buckling stress is simply given by the Euler buckling stress:

$$
\sigma_{\text {buckle }}=\frac{\pi^{2} \mathrm{E}_{\mathrm{f}}^{\prime}}{3}\left(\frac{h}{a}\right)^{2}
$$

Figure 3. PLST strain energy release rate as a function of the crack length.

Although the strain energy release rate can be calculated at any point before buckling based on the load data from equation (6), G prior to crack onset does not have any physical meaning, since the crack does not grow, and no energy is

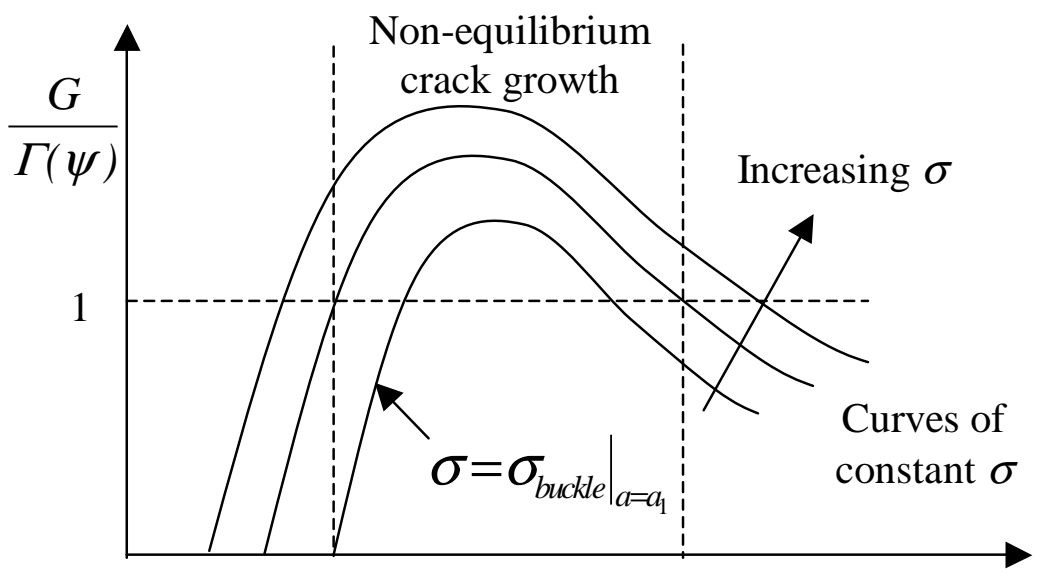

$\mathrm{a}_{1}$ being released yet. The minimum $\mathrm{G}$ measured would be $18 \mathrm{~J} / \mathrm{m}^{2}$ corresponding to $10 \mathrm{~mm}$ crack length (Table 1$)$. It is difficult to detect the crack initial propagation point. But this may be the way to determine the critical strain energy release rate.

Figure 4. Interpretation of the unstable crack growth during buckling for the PLST.

\section{FINITE ELEMENT MODELING}

The PLST prior to buckling have been modeled using finite element analysis, employing PATRAN and ABAQUS commercial codes. The finite element model of the fine line and substrate is shown in Figure 5. Finer meshes were used around the crack tip. The strain singularity at the bimaterial crack tip is $\mathrm{r}^{-1 / 2+i \varepsilon}$. Therefore quarter-point-node elements were used 
at the crack tip to simulate the dominant square root singularity $\left(\mathrm{r}^{-1 / 2}\right)$. The crack-tip mesh is also shown in Figure 5, where 3604 elements and 11120 nodes are generated in the model. The deformed line is shown in Figure 7, where the scale represents absolute deflection in microns.

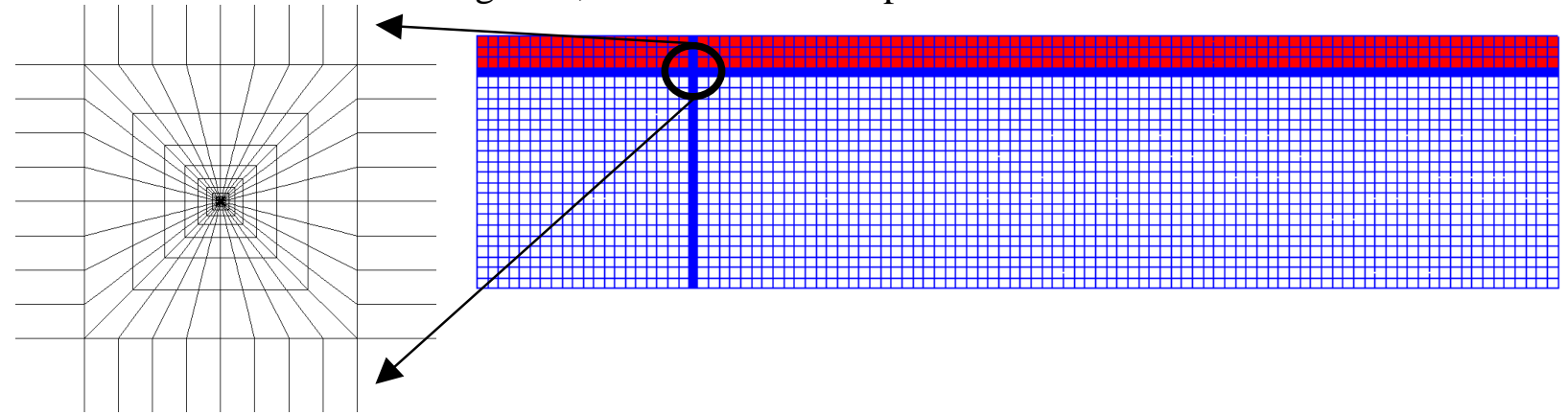

Figure 5. FEM model of the PLST with finer mesh around the crack tip.

Table 1. FEM calculations based on the experimental data.

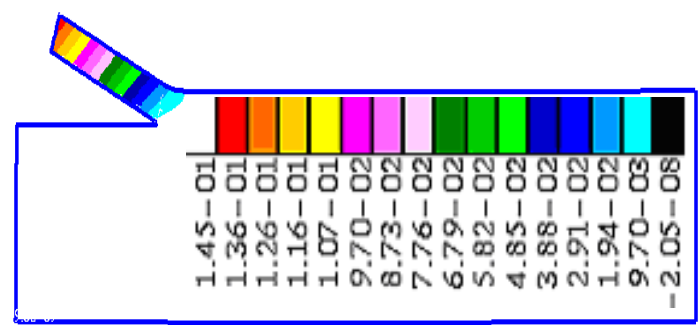

Figure 6. Finite element model of the deformed line.

\begin{tabular}{|r|l|r|r|r|}
\hline $\begin{array}{l}\text { Crack } \\
\text { Length } \\
(\mathrm{mm})\end{array}$ & $\begin{array}{l}\text { Load } \\
(\mathrm{N})\end{array}$ & $\begin{array}{l}\text { Numerical } \\
\mathrm{G}\left(\mathrm{J} / \mathrm{m}^{2}\right)\end{array}$ & $\begin{array}{l}\text { Phase } \\
\text { Angle, } \\
\text { degrees }\end{array}$ & $\begin{array}{l}\text { Analytical } \\
\mathrm{G}\left(\mathrm{J} / \mathrm{m}^{2}\right)\end{array}$ \\
\hline 10.000 & 224.61 & 13.60 & 45.0 & 18.368 \\
\hline 10.278 & 546.88 & 100.5 & 44.1 & 108.89 \\
\hline 13.368 & 991.21 & 331.6 & 44.1 & 357.72 \\
\hline 16.499 & 1303.7 & 572.5 & 44.0 & 618.83 \\
\hline 18.356 & 1499.0 & 758.5 & 44.0 & 818.14 \\
\hline 23.678 & 1621.1 & 891.7 & 43.99 & 956.82 \\
\hline
\end{tabular}
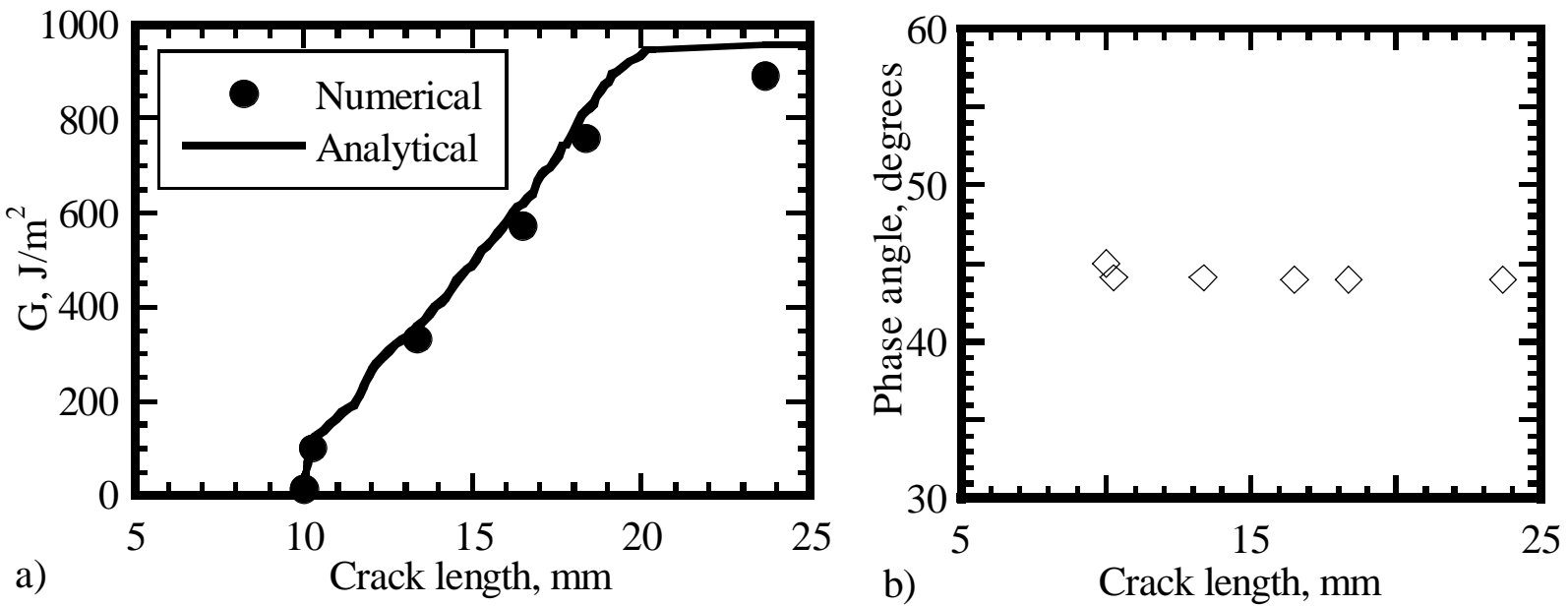

Figure 8. a) FEM calculations comparison to the analytical data; b) Phase angle as a function of the crack length.

Strain energy release rate has been calculated based on the experimental load and crack length data. Six data points have been chosen. FEM calculations were shown in Figure 8a with 
the closed form solution (equation (6)) results. The difference between the numerical results and the analytical results is within $8 \%$.

It is clear that the energy release rate increases significantly as the crack grows. To understand whether this increase is due to the change in mode mixity, the loading phase angle was also calculated at each point (Figure $8 \mathrm{~b}$ ). Phase angle is almost constant at $45^{\circ}$, decreasing with the crack length. This indicates that the fine line scratch tests could provide a consistent loading phase angle of $45^{\circ}$ for the polycarbonate/steel combination. It also indicates that the increase in strain energy release rate is not due to the phase angle increase, but rather due to the increasing crack tip plastic energy dissipation contributions.

\section{SUMMARY}

Macroscopic experiments with the macroscopic Precracked Line Scratch Test have provided the crack length measurements, which allowed constructing the strain energy release rate curve throughout the whole test, prior and after buckling. Finite element analysis has also confirmed the validity of a simple mechanics approach before buckling. FEM showed almost no phase angle change with the crack length prior to buckling.

With the analysis in place, the precracked line scratch test can be used conveniently to study the adhesion of interconnects and passives, MEMS devices and packages on different scales.

\section{ACKNOWLEDGEMENTS}

Authors would like to acknowledge support for this research from John C. Nelson, David F. Bahr and Michael D. Kriese.

\section{REFERENCES}

1. M.P. de Boer, M. Kriese, W.W. Gerberich, J. Mater. Res. 12(10), 1997, 2673-2685

2. A.A. Volinsky, J.C. Nelson, W.W. Gerberich, Mat. Res. Soc. Symp. Proc. Vol. 563, 1999

3. A. Pocius, "Adhesion and Adhesives Technology. An Introduction." Hanser Publishers, 1997

4. M. Ohring, "The Materials Science of Thin Films", Academic Press Inc. 1991, p. 444

5. W.T. Chen, T.F. Flavin, IBM J. Res. Develop. 16, pp.203-213, 1972

6. A. Bagchi, A. Evans, MRS Symp. Proc. Vol. 383, pp. 183-197, 1995

7. M.L. Jokl, V. Vitek, C.J. McMahon, Acta Metal., 28, p. 1479, 1980

8. M.P. De Boer, J.C. Nelson and W.W. Gerberich, J. Mater. Res., 13(4), 1002-1014, 1998

9. M.P. De Boer, Ph.D. Dissertation, University of Minnesota, 1996

10. M.P. De Boer and W.W. Gerberich, Acta Mater. 44 No 8, pp. 3169-3175, 1996

11. R.W. Hertzberg, Deformation and Fracture Mechanics of Engineering Materials, John Wiley \& Sons, Inc., p. 338, 1996

12. J. Hutchinson and Z. Suo, Advances in Applied Mechanics, Vol. 29, pp. 63- 191, 1992 